\title{
Branchial Cleft Cyst Mimicking Malignant Thyroid Neoplasm Concurrent with Medullary and Papillary Thyroid Carcinoma: a Case Report
}

\author{
Wook $\mathrm{Yi}^{1}$, Bo Hyun Kim ${ }^{1}$, Byung Joo Lee ${ }^{2}$ and Kyungbin Kim ${ }^{3}$ \\ Division of Endocrinology and Metabolism, Department of Internal Medicine, Biomedical Research Institute, Pusan National \\ University Hospital ${ }^{1}$, Department of Otorhinolaryngology Head and Neck Surgery, Pusan National University Hospital ${ }^{2}$, \\ Department of Pathology, Pusan National University Hospital ${ }^{3}$, Busan, Korea
}

\begin{abstract}
Branchial cleft cysts are the most common lateral neck mass, and usually have well-delineated cystic structures under ultrasonography. Ultrasonography and fine needle aspiration cytology is very helpful during preoperative diagnosis. However, these cysts may occur in unusual locations and have a sonographic morphology similar to malignant thyroid neoplasm. A 61-year-old woman presented with thyroid nodules, and ultrasonography revealed a $0.8-\mathrm{cm}$, hypoechoic, solid nodule with microcalcifications in the right lobe of thyroid in the lower portion. Postoperative histopathological examination revealed the $0.8-\mathrm{cm}$ hypoechoic nodule to be a branchial cleft cyst. In addition, concurrent medullary thyroid carcinoma (MTC) and papillary thyroid carcinoma (PTC) were found. Herein, we present a rare case of branchial cleft cyst mimicking malignant thyroid neoplasm concurrent with medullary and PTC.
\end{abstract}

Key Words: Branchial cleft cyst, Papillary thyroid carcinoma, Medullary thyroid carcinoma, Ultrasonography

\section{Introduction}

Branchial cleft cysts are derived from remnants of the branchial apparatus and most commonly occur in the lateral neck region along the anterior border of the sternocleidomastoid muscle in the upper third of the neck. ${ }^{1)}$ However, there are previous reports documenting branchial cleft cysts at unusual sites or organs, such as the oral cavity, parotid gland, and thyroid gland. ${ }^{2-4)}$ Among its different types, the third and fourth branchial cleft cysts are situated adjacent to the thyroid gland and have been rarely reported. ${ }^{5,6)}$

Ultrasonography (US) is a standard tool used to evaluate thyroid nodules and branchial cleft cysts.
Generally, branchial cleft cysts are visualized as well-delineated hypoechoic cystic structures with posterior acoustic enhancement under US. However, their echogenicity can vary, causing them to appear septated, inhomogeneous, and even solid. ${ }^{78)}$ Fine needle aspiration (FNA) can be performed in indeterminate cases, such as during the presence of a solid component, or during instances of internal doppler signals, for a differential diagnosis.

Co-existing medullary thyroid carcinoma (MTC) and papillary thyroid carcinoma (PTC) in the thyroid gland is rare. Despite the differences in cell origins and histopathological features of MTC and PTC, they may coincide within the same tumor or may be localized in the same thyroid tissue. Concurrent MTC and PTC

Received January 28, 2021 / Accepted February 2, 2021

Correspondence: Bo Hyun Kim, MD, PhD, Division of Endocrinology and Metabolism, Department of Internal Medicine, Biomedical Research Institute, Pusan National University Hospital, 179 Gudeok-ro, Seo-gu, Busan 49241, Korea Tel: 82-51-240-7236, Fax: 82-51-254-3237, E-mail: pons71@hanmail.net 
represents less than $1 \%$ of all thyroid malignancies. ${ }^{9}$

To best our knowledge, there was no previous reports of branchial cleft cyst with concurrent MTC and PTC. Here, we report a rare case of branchial cleft cyst mimicking a primary thyroid neoplasm in a patient with concurrent MTC and PTC.

\section{Case Report}

A 61-year-old woman presented with incidental thyroid nodules discovered on a US scan during health screening at our Endocrinology and Metabolism Department's outpatient clinic. The patient did not have any symptoms associated with the mass such as dyspnea, dysphagia, or hoarseness of voice. The patient also had no history of previous neck irradiation or a family history of thyroid cancer. No palpable mass or swelling of the neck area was detected during physical examination. Laboratory data reported all thyroid function test results to be within normal limits. However, a thyroid US revealed a $0.8-\mathrm{cm}$ hypoechoic nodule with ill-defined margins and microcalcifications in the lower portion of the right thyroid gland (Fig. 1A) and an oval $0.6-\mathrm{cm}$ hypoechoic nodule with well-defined margins in the upper portion of the right thyroid gland (Fig. 1B). FNA was performed for the $0.8-\mathrm{cm}$ hypoechoic nodule, and cytological examination re- vealed no evidence of malignancy. Thus, we decided to evaluate the thyroid nodules by regular sonography follow-up. Two years later, the size of the $0.8-\mathrm{cm}$ nodule increased upon US (Fig. 1C, D), and the FNA was hence repeated. The cytological examination revealed the presence of mature squamous cells.

A right hemithyroidectomy was performed to exclude the possibility of malignancies. The $0.8-\mathrm{cm}$ nodule, which was located in the right lower portion of the thyroid gland during US, was found to be attached to the inferior margin of the right thyroid gland and not within the thyroid gland. Postoperative histopathological examination confirmed the diagnosis of a branchial cleft cyst. Grossly, a $0.5 \times 0.3 \times 0.2-\mathrm{cm}$ sized relatively well-defined yellowish tan nodule was found in the upper portion of the right thyroid gland and it was diagnosed as MTC. Additionally, a $0.1-\mathrm{cm}$ sized PTC, not grossly visible, was incidentally found in the middle portion of the right thyroid gland under microscopy (Fig. 2). Upon histopathological examination, the branchial cleft cyst was lined by stratified squamous epithelium without adnexal structures and the contents of the cyst were keratin debris (Fig. 3A). The tumor cells in the MTC nodule had a round to plasmacytoid appearance and were intensely positive for calcitonin (Fig. 3B, C). Nuclei displaying variations in size and shape, membrane irregularity, nuclear longitudinal
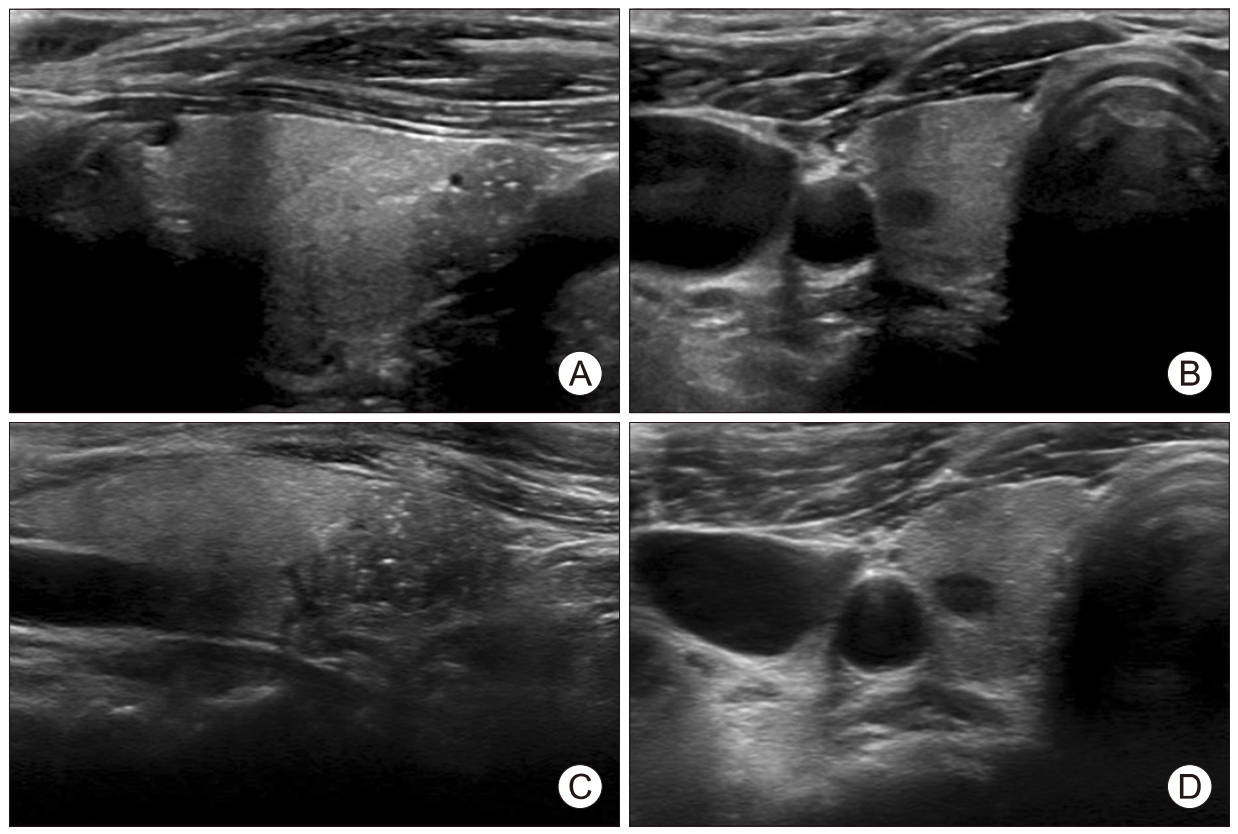

Fig. 1. (A) Initial ultrasonography (US) of branchial cleft cyst, (B) initial US of medullary thyroid carcinoma. (C) Follow-up US of branchial cleft cyst. (D) Followup US of medullary thyroid carcinoma. 

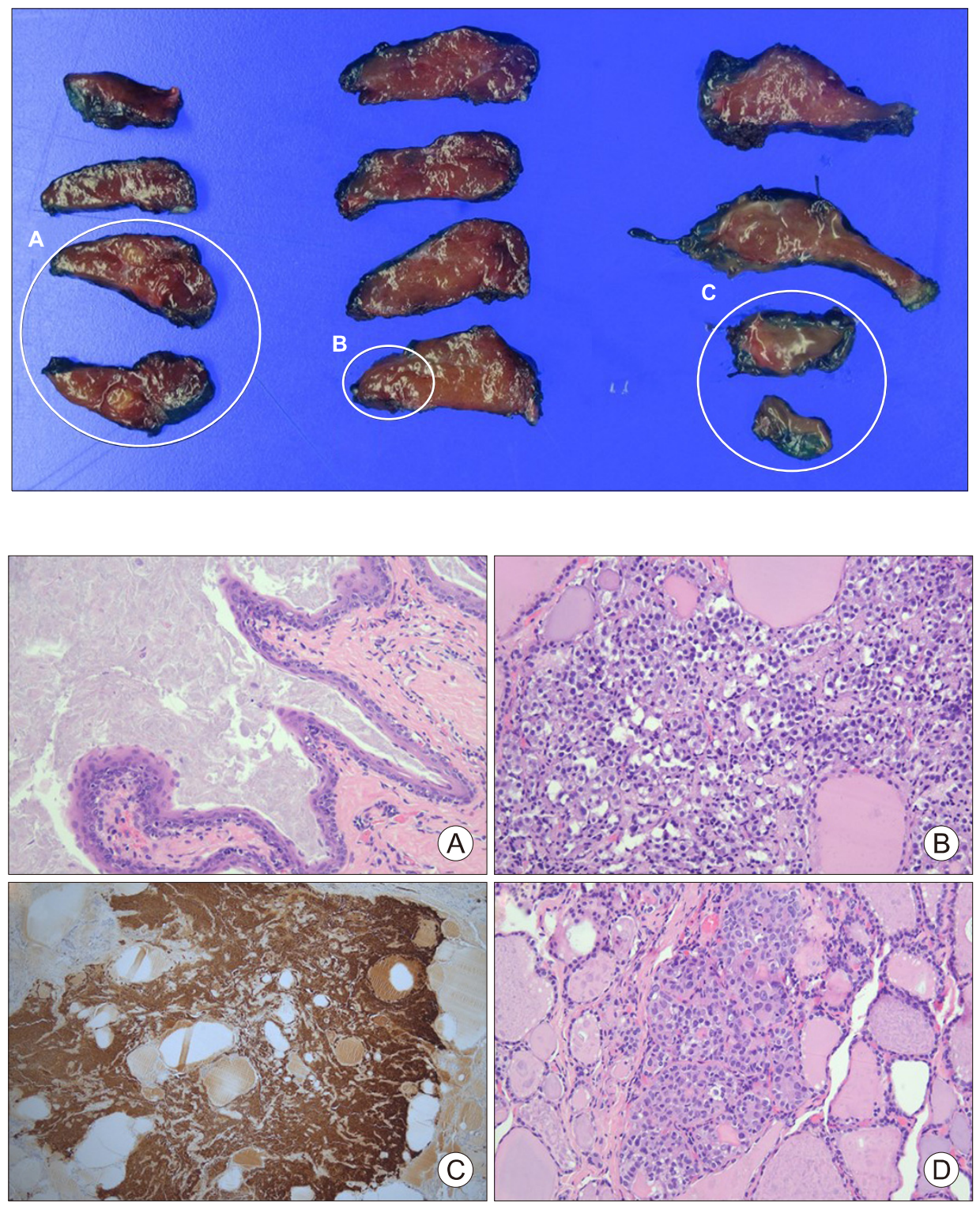

Fig. 2. The cut surface of the right thyroid gland. (A) Medullary thyroid carcinoma, (B) papillary thyroid carcinoma, (C) branchial cleft cyst.

Fig. 3. Postoperative histopathological examination of branchial cleft cyst, medullary thyroid carcinoma and papillary thyroid carcinoma. (A) Branchial cleft cyst, $\mathrm{He}^{-}$ matoxylin and Eosin (H\&E) staining, magnification $\times 200$, (B) medullary thyroid carcinoma, H\&E staining, magnification $\times 200$, (C) positive calcitonin immunostaining in medullary thyroid carcinoma, (D) papillary thyroid carcinoma, H\&E staining, magnification $\times 200$. grooves, and pseudo-inclusions were found in the middle portion of the right thyroid gland, and these characteristics were representative of a PTC lesion (Fig. 3D).

There was no history of familial MTC in the patient or the family, with no evidence of multiple endocrine neoplasia type 2. Thus, this MTC case was classified as sporadic MTC. We further evaluated serum calcitonin and imaging studies such as chest computed tomography and thyroid US. The serum calcitonin level was $2.76 \mathrm{pg} / \mathrm{mL}$, which was within the normal range, and there was no residual MTC during US. No metastatic lesions were noted on the chest computed tomography. Therefore, completion thyroidectomy have not been recommended. After 3 months of follow-up, there was no evidence of recurrence.

\section{Discussion}

In this case study, we reported a very rare case of branchial cleft cyst with unusual sonographic morphology, unique location, and an incidental concurrence of MTC and PTC diagnosed post-hemithyroidectomy. Such unusual findings during US and FNA can be a diagnostic challenge for clinicians and require a deeper understanding of the nature of the neck masses and 
thyroid nodules.

Branchial cleft cysts are one of the most common congenital neck masses, which are present on the lateral aspect of the neck. ${ }^{6}$ ) Third and fourth branchial cleft cysts, although rarely encountered, account for $3-10 \%$ of all branchial lesions and are usually located in close contact with the left thyroid gland. ${ }^{10)}$ Additionally, these are associated with recurrent neck abscesses or a preceding thyroiditis. For diagnosis, sonography and cytological findings of FNA serve as important tools in branchial cleft cyst for differential diagnosis of other neck masses. Typical US characteristics include a well-defined cystic mass with an enhanced rim. ${ }^{6)}$ The branchial cleft cyst in this case presented a solid hypoechoic nodule with microcalcifications. Also, it was not clear whether the cyst was located inside or outside the thyroid gland. Due to its unusual US characteristics, the nodule was thought to be located within the thyroid gland. The cytological result of the FNA revealed mature squamous cells. Mature squamous cells in thyroid FNAs can represent a variety of thyroid disorders, such as branchial cleft cysts, epidermoid cysts, thyroglossal duct remnants, or malignant thyroid lesions, but are mostly benign. ${ }^{11)}$ The FNA result suggested that the nodule was a benign cyst, such as a branchial cleft cyst; however, the US characteristics and increased size suggested that the nodule is possibly malignant. Therefore, we discussed the management of the nodule with the patient, who agreed to the surgical resection. After right hemithyroidectomy, the branchial cleft cyst was diagnosed with concurrent MTC and PTC, and further evaluation was required to diagnose metastasis.

MTC with concurrent PTC was first reported by Lamberg et al. ${ }^{12)}$ in 1981. The co-existence of MTC and PTC can happen in two different forms. The first involves a discrete MTC and PTC separated by normal thyroid tissue, whereas the second involves a mixed medullary and follicular-derived thyroid carcinoma, according to the World Health Organization classification system. ${ }^{13)}$ Our case belongs to the first category. Generally accepted hypotheses for co-occurrence of MTC and PTC are the common stem cell theory, hostage hypothesis, field effect theory, and collision theory. ${ }^{14,15)}$ The collision theory suggests that two independent tumors are located in the same lesion by simple coincidence, and most reported cases could be explained by this theory. ${ }^{15)}$ Furthermore, Kim et al. ${ }^{15)}$ showed that PTC with co-existing MTC was less than $1.0 \mathrm{~cm}$ in almost all cases, and other reports showed that approximately $77 \%$ of the papillary carcinomas presented as papillary thyroid microcarcinoma. ${ }^{15,16)}$ In our case, the PTC was $0.6 \mathrm{~cm}$ in size, which was consistent with that in the previous reports. A recent epidemiologic study showed that MTC had more cases of advanced stage presentations and a higher mortality than PTC; therefore, the management of MTC was suggested to be prioritized in concurrent MTC and PTC. ${ }^{17)}$ According to the revised American Thyroid Association guideline, completion thyroidectomy following hemithyroidectomy is not recommended unless the patient has significantly elevated basal or stimulated serum calcitonin levels postoperatively, or unless imaging studies show residual MTC for sporadic MTC. ${ }^{18)}$ The limitation in this case is that preoperative measurement of serum calcitonin concentration was not performed. During preoperative evaluation, MTC was not considered, thus only thyroid function test and serum thyroglobulin were measured. However the MTC in our case was considered sporadic, and postoperative evaluation showed no residual tumor and normal serum calcitonin levels. Completion thyroidectomy was therefore not indicated, and no evidence of MTC recurrence was observed upon follow-up.

In conclusion, we found a brachial cleft cyst mimicking a malignant thyroid neoplasm during US in a patient with concurrent MTC and PTC, diagnosed post-operatively. Branchial cleft cysts generally present under US as lateral neck masses with a well-delineated cystic structure. However, branchial cleft cysts may have various sonographic appearances and can be located inside or adjacent to the thyroid gland. Therefore, physicians should consider malignant thyroid neoplasms as a differential diagnosis for these lesions and need to comprehend the nature of these lesions. Furthermore, physicians should also consider whether branchial cleft cysts may co-occur with thyroid carcinomas during preoperative and postoperative 
examinations because MTC and PTC may incidentally co-exist with branchial cleft cysts.

\section{Acknowledgments}

This work was supported by clinical research grant from Pusan National University Hospital in 2020.

\section{Conflicts of Interest}

No potential conflict of interest relevant to this article was reported.

\section{Orcid}

Wook Yi: https://orcid.org/0000-0001-6519-9397

Bo Hyun Kim: https://orcid.org/0000-0001-9632-9457

Byung Joo Lee: https://orcid.org/0000-0001-7091-6688

Kyungbin Kim: https://orcid.org/0000-0001-5430-4235

\section{References}

1) Maran AG, Buchanan DR. Branchial cysts, sinuses and fistulae. Clin Otolaryngol Allied Sci 1978;3(1):77-92.

2) Giunta J, Cataldo E. Lymphoepithelial cysts of the oral mucosa. Oral Surg Oral Med Oral Pathol 1973;35(1):77-84.

3) Weidner N, Geisinger KR, Sterling RT, Miller TR, Yen TS. Benign lymphoepithelial cysts of the parotid gland. A histologic, cytologic, and ultrastructural study. Am J Clin Pathol 1986; 85(4):395-401.

4) Miyazaki M, Kiuchi S, Fujioka Y. Branchial cleft-like cysts in Hashimoto's thyroiditis: a case report and literature review. Pathol Int 2016;66(5):297-301.

5) Thomas B, Shroff M, Forte V, Blaser S, James A. Revisiting imaging features and the embryologic basis of third and fourth branchial anomalies. AJNR Am J Neuroradiol 2010;31(4):75560.
6) Bagchi A, Hira P, Mittal K, Priyamvara A, Dey AK. Branchial cleft cysts: a pictorial review. Pol J Radiol 2018;83:e204-e9.

7) Gritzmann N, Hollerweger A, Macheiner P, Rettenbacher T. Sonography of soft tissue masses of the neck. J Clin Ultrasound 2002;30(6):356-73.

8) Brown RE, Harave S. Diagnostic imaging of benign and malignant neck masses in children-a pictorial review. Quant Imaging Med Surg 2016;6(5):591-604.

9) Sizemore GW. Medullary carcinoma of the thyroid gland. Semin Oncol 1987;14(3):306-14.

10) Liberman M, Kay S, Emil S, Flageole H, Nguyen LT, Tewfik TL, et al. Ten years of experience with third and fourth branchial remnants. J Pediatr Surg 2002;37(5):685-90.

11) Gage H, Hubbard E, Nodit L. Multiple squamous cells in thyroid fine needle aspiration: friends or foes? Diagn Cytopathol 2016;44(8):676-81.

12) Lamberg BA, Reissel P, Stenman S, Koivuniemi A, Ekbolm $\mathrm{M}$, Makinen J, et al. Concurrent medullary and papillary thyroid carcinoma in the same thyroid lobe and in siblings. Acta Med Scand 1981;209(5):421-4.

13) Hedinger $\mathrm{C}$, Williams ED, Sobin LH. The WHO histological classification of thyroid tumors: a commentary on the second edition. Cancer 1989;63(5):908-11.

14) Dikbas O, Duman AA, Guvendi GF. Medullary thyroid carcinoma and papillary thyroid carcinoma in the same patient as a collision tumour. Case Rep Endocrinol 2019;2019:4038628.

15) Kim WG, Gong G, Kim EY, Kim TY, Hong SJ, Kim WB, et al. Concurrent occurrence of medullary thyroid carcinoma and papillary thyroid carcinoma in the same thyroid should be considered as coincidental. Clin Endocrinol (Oxf) 2010;72(2): 256-63.

16) Adnan Z, Arad E, Dana J, Shendler Y, Baron E. Simultaneous occurrence of medullary and papillary thyroid microcarcinomas: a case series and review of the literature. J Med Case Rep 2013;7:26.

17) Appetecchia M, Lauretta R, Barnabei A, Pieruzzi L, Terrenato I, Cavedon E, et al. Epidemiology of simultaneous medullary and papillary thyroid carcinomas (MTC/PTC): an Italian multicenter study. Cancers (Basel) 2019;11(10):1516.

18) Wells SA Jr, Asa SL, Dralle H, Elisei R, Evans DB, Gagel $\mathrm{RF}$, et al. Revised American Thyroid Association guidelines for the management of medullary thyroid carcinoma. Thyroid 2015;25(6):567-610 